\title{
IMAGE PROCESSING BASED ON A MODEL OF THE MAMIMALIAN RETINA
}

\author{
J.A. Martín-Pereda and A. González-Marcos \\ ETS Ingenieros de Telecomunicación \\ Universidad Politécnica de Madrid. Ciudad Universitaria \\ 28040 Madrid. Spain
}

\begin{abstract}
A first study in order to construct a simple model of the mammalian retina is reported. The basic elements for this model are Optical Programmable Logic Cells, OPLCs, previously employed as a functional element for Optical Computing. The same type of circuit simulates the five types of neurons present in the retina. Different responses are obtained by modifying either internal or external connections. Two types of behaviors are reported: symmetrical and non-symmetrical with respect to light position. Some other higher functions, as the possibility to differentiate between symmetric and non-symmetric light images, are performed by another simulation of the first layers of the visual cortex. The possibility to apply these models to image processing is reported.
\end{abstract}

Keywords: Retina Modelling, Image Processing, Optical Information Processing, Vision, Biological Neural

Networks

\section{INTRODUCTION}

The mammalian retina, as well as many other sensorial systems in living bodies, offers a large variety of responses to external stimuli. These responses, after being analyzed by higher brain levels, determine the animal behaviour choosing one interpretation among other different possibilities. This way of working gives the opportunity to extract some solutions to problems reported in many other fields, ranging from consumer photonics to industrial systems. In our present case, the mammalian retina constitutes a good place to learn how to analyse any type of images. The reason is the large number of functions carried out by it. Moreover, the form of processing images in parallel is superior to the way in which it is done by most of the present artificial vision systems. To try to obtain some lessons from the way the retina works can be a very useful task.

Most of the operations handled by the biological retina are performed with very sophisticated hardware. No software is present. The working architectures are based on the use of a very high number of neural circuits. Their basic element, the neurones, presents very peculiar behaviours. Each of them has its own environment. Two similar neurones could give rise to different outputs depending on the way their synapses are arranged. These outputs will determine, on their turn, the processes in the following stages.

The aim of this work is to offer a possibility to image processing based on a model of the mammalian retina and some other higher levels of the visual cortical system.

\section{BASIS OF THE MODEL}

The basis of our model is a simple structure, shown in Fig. 1, based on a model previously given by Dowling ${ }^{1}$. It is composed by the five types of cells appearing at the mammalian retina, namely, photoreceptors, horizontals, bipolars, amacrine and ganglion cells. They are arranged into three layers and they have different responses depending on the characteristics of the impinging light at the receptors. In the case shown in Fig 1, where just light is on one of the photoreceptors, a particular set of different responses is shown at the insets of each cell. Although the real responses at the biological cells have a partially analogic character in our model they have been converted to digital signals. This has been due to the easier way that they can been implemented. As it can be seen, the responses at the three adopted ganglion cells outputs are of three different types. The first one gives a regular train of pulses when here is no light on the receptors and stops this train during the time interval when there is light. The second one gives a short pulse at the beginning and at the end of the input light signal. Finally, the third one just offers a train of pulses during the time when there is light at receptor $\mathrm{R}_{1}$. This configuration, as it will be shown later, offers the possibility to detect several characteristics of the input light, for instance, if it comes from right to left or viceversa. 


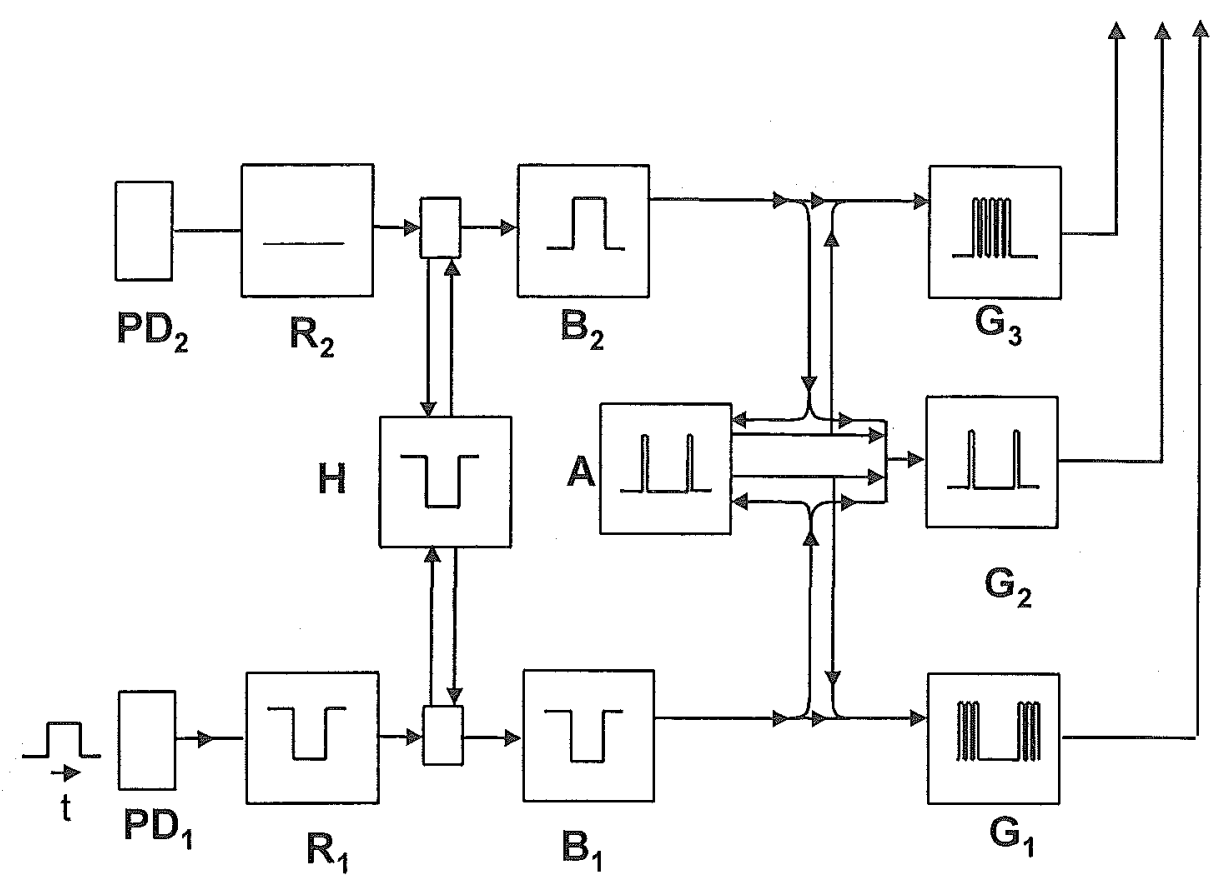

Fig. 1 Retina model. This configuration summarises the main activity of the different retinal cells. $P D_{1}, P D_{2}-$ Photodetectors; $R_{1}, R_{2}$ - Receptor cells; $H$ - Horizontal cell; $B_{1}, B_{2}$ - Bipolar cells; A - Amacrine cell; $G_{1}, G_{2}$ and $G_{3}$ Ganglion cells. Some of the possible responses of the different cells are given at the insets.

In order to implement each one of the cells in the retina structure, a configuration as the one shown in Fig. 2 has been adopted. Two different types of non-linear devices compose it. Q-device is a thresholding or switching on-off structure offering a logic "zero" output up to a fixed value of the input and a logic "one" from this value on. P-device is a multistate device with a behaviour similar to the offered by SEED devices, namely an $\mathrm{N}$-like shape in the output versus input characteristic. We have employed this structure previously as the main block for certain parts of an optical computer architecture ${ }^{2-4}$. It received the name of Optically Programmable Logic Cell (OPLC) there. A brief description on its method of operation, as well as the way it has been implemented, can be seen there. The output of each of the non-linear devices corresponds to the two final outputs, $\mathrm{O}_{1} \mathrm{y} \mathrm{O}_{2}$, of the cell. The possible inputs to the circuit are four. Two of them are for the input data, $\mathrm{I}_{1}$ and $\mathrm{I}_{2}$, and the other two, $g$ and $h$, for control signals. The way these four inputs are distributed inside the circuit it is also represented in Figure 2. The corresponding inputs to the non-linear devices are based on these signals. The characteristics of the non-linear devices are shown in insets of Figure 2. The functions that have been obtained from this structure are fourteen pairs of logic functions. Details are given in ${ }^{2}$.

The adopted architecture has the configuration seen in Fig. 1. Each block has an internal structure similar to the one shown in Fig. 2. In most of the cases, just one or two inputs receive signals from previous layers. Other inputs get no signals. With respect to outputs, just one of the two possible ones is connected to the following cell. This situation gives the opportunity to employ the non-operative inputs and outputs in other possible processes. We have adopted here the simplest structure and maintain other possibilities for further works.

According to the photoreceptors way of working, we have adopted, in most of the cases, the level of a logic " 1 " as the resting potential when there is no light going on the photoreceptors and a logic "0" when an optical signal is present. Because of the character of our present model, there is no possibility to register different light levels. A possibility to introduce this parameter comes from the multilevel characteristics of the employed signal. This possibility deserves a further study.

The above situation means that the receptor cells must operate with a NAND function. And so the OPLC was programmed. In our model, when there is some light acting on the receptor $\mathrm{R}_{1}$, it switches from " 1 " to " 0 ". No light in receptor $\mathrm{R}_{2}$ implies to keep the " 1 " level.

The photoreceptor response goes then to the horizontal and bipolar cells. The horizontal cell performs a NAND function. In bipolar cells, $B_{1}$ and $B_{2}$, the situation is somehow different in both of them. Dowling's model gives a hyperpolarization response for $B_{1}$ and a depolarization for $B_{2}$. This response is obtained with AND and NAND functions on cells $B_{1}$ and $B_{2}$, respectively. This behavior can be achieved with two different configurations of the OPLC. These 


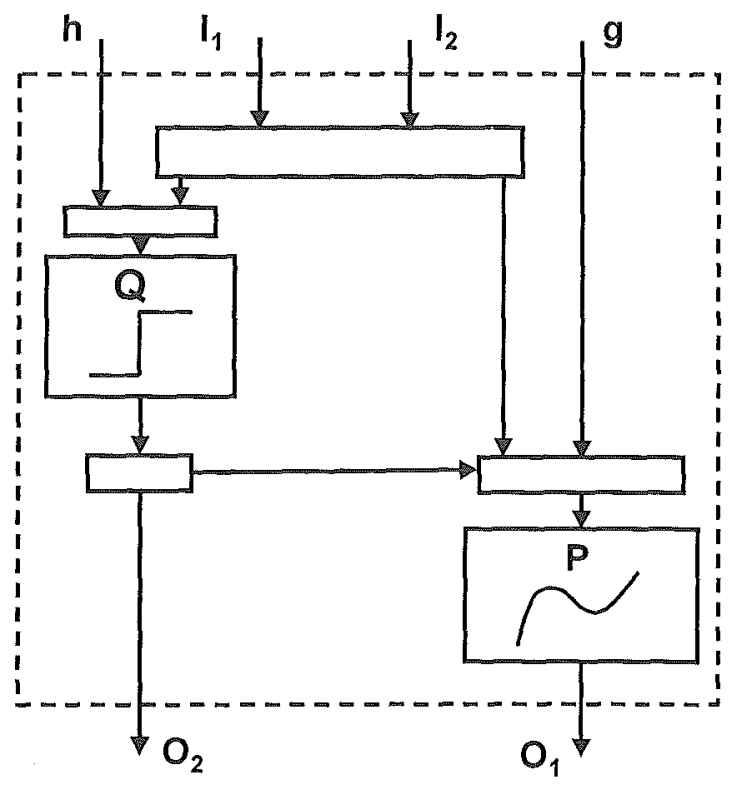

configurations do not correspond to changes in the internal structure but on the way the connections are taken. This means adopting a particular arrangement for how data and control signals reach the inputs of the OPLC.

There are several possible outputs obtained from amacrine and ganglion cells. Therefore, just two cases have been studied. The first one corresponds to a non-symmetrical way of working. Namely, light on receptor $R_{1}$ and no light on $R_{2}$ gives outputs at ganglion cells, $G_{1}, G_{2}$ and $G_{3}$, different from when there is no light at $R_{1}$ and light at $R_{2}$. The second case we have studied is the symmetrical one. If light is on $R_{1}$ and no light on $R_{2}$, outputs at ganglion cells are similar than when the light is on $R_{2}$ and no light on $R_{1}$.

Fig. 2 Optical Programmable Logic Cell -OPLC- acting as a Neuron Cell: Internal structure. $P$ and $Q$ are non-linear logic devices.

The first situation could differentiate the sense of motion from right to left and viceversa. The second one would differentiate the presence of light on some part of the visual receptor system.

\subsection{First case: asymmetrical behavior}

The main objective of this configuration is the detection of sense in light motion. The adopted architecture has similar signals in the neurons to the ones shown in the Dowling model. Hence, amacrine cell must give a different output signal than the ones appearing at receptor, horizontal and bipolar cells. Its resting state is, in our case, a logic "1" and switches to a logic " 0 " at the beginning and at the end of light pulses. This switch is in the form of a brief impulse. After this peak, it returns to the resting state. Hence, its behavior is in the form of action potentials with a burst of just one pulse.

An important point to be said here is that, with this arrangement, the amacrine is in charge of detecting the illumination time on receptors. This aspect is not considered in the literature. The more important function customarily assigned to amacrine cells corresponds to spatial aspects of visual signals. We believe that this proposed temporal aspect needs to be considered in future works.

The composite signals from bipolar and amacrine cells arrive to the ganglion cells from where the final signal is obtained. There exits several possible responses from the ganglion cells. Three of them are In Fig. 1 . The first one is a train of pulses, its length being the same as that of the input flash. The third one is always a sequence of short pulses; only when it is light at the input, the pulses disappear. Finally, the second one shows a continuous train of pulses. It disappears just at the beginning and at the end of the initial flash.

\subsection{Second case: symmetrical behavior}

The function of this structure can be, for instance, the detection of light motion or the spatial extension of any image. There is no difference at the ganglion signals if light is on the right receptor or in the left one: ganglion outputs are the same in both cases. In our present case, where just two receptors have been taken, the second above-mentioned application of this circuit is less evident. But it can be obtained for a larger number of receptors.

Ganglion responses are different here from the previous one. The ganglion cell of the channel with light responds now with a pulse when the input is applied. On the contrary, the ganglion cell corresponding to the channel that gets no light, offers an output composed by action potentials when there is light and a " 0 " level when there is no light. Ganglion cell in between offers a similar behavior to this last neuron, but with resting voltage of "1" instead "0". Similar behavior occurs when light signal is interchanged. 


\section{MODEL SIMULATION}

To develop a first study of the model feasibility, a computer simulation has been adopted. The external connections for the OPLCs are the same in both reported behaviours. MATLAB tools have been employed.

There are two data signals to be applied to photoreceptors: a constant background signal and a pulses train. The second one represents short light flashes whereas the former one corresponds to the ambient light.

A gain constant in each channel, before the input of each cell, is added. Although it is not necessary in our present study, it has been added in order to maintain the possibility of incorporating a weight to the signals. This fact should be considered when higher level tasks would be added to the system.

As it can be seen, in Fig.1, the horizontal cell is fed by both receptors. The information is then transferred to the bipolar cells of each channel. Therefore, bipolar cells process the horizontal plus his corresponding receptor information. In the same way, the amacrine cell processes the signal obtained from the two bipolar cells. The result will be part of the input to the ganglion layer. The three different ganglion cells in this layer have responses depending on their inputs. The results obtained for the first configuration correspond to the ganglion cell behavior of the Dowling model. They are shown in Fig. 3. The coding for polarization and depolarization is the one explained previously. We obtain the results expected using the same basic block for all the neurones at the retina layers.
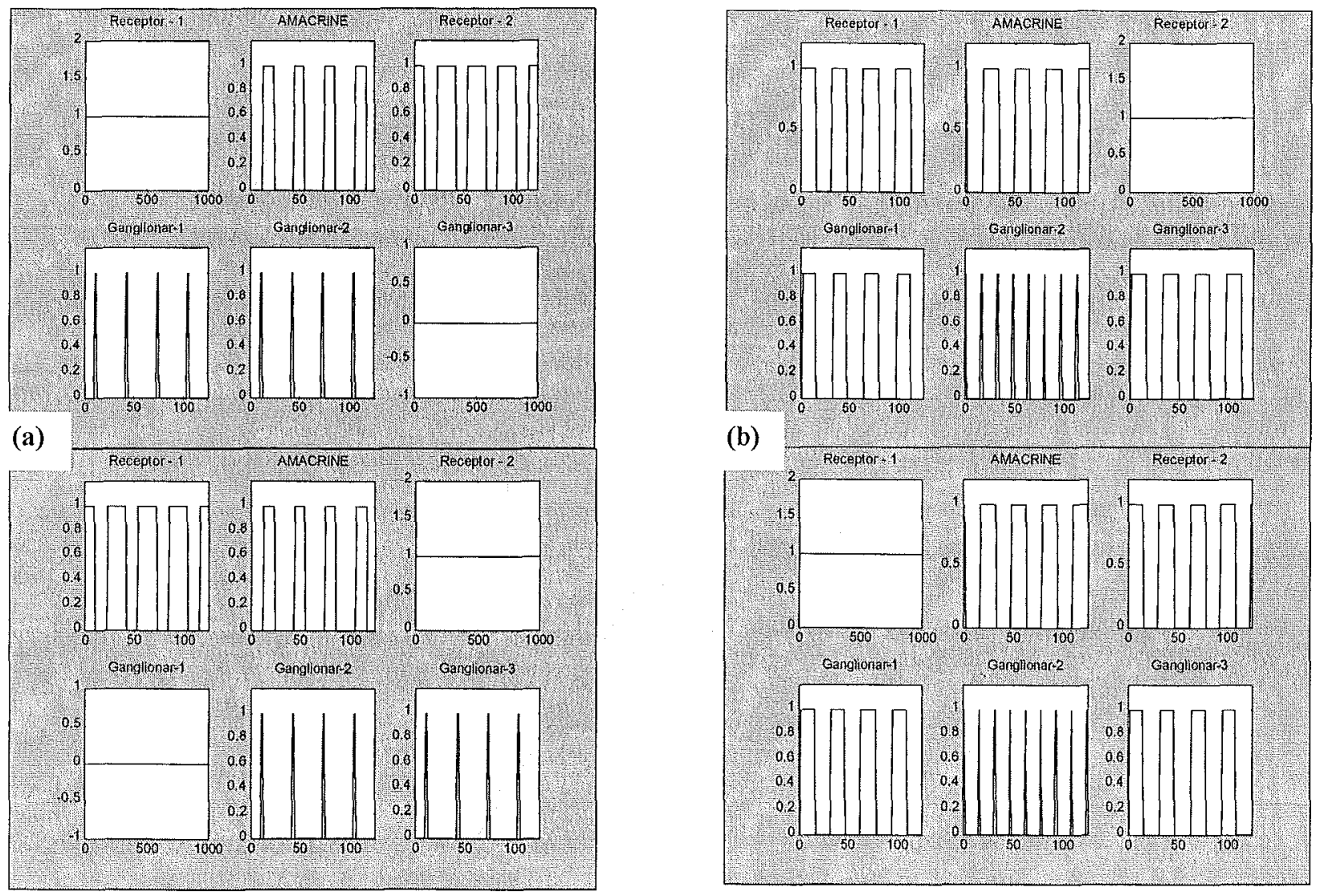

Fig. 3.- a) Retinal neural responses for the asymetric behaviour. b) Ibid. for the symetrical case.

This first structure has the characteristic of being non-symmetric. This means that the behaviour of the total retina layer is not the same if the light is coming from the right path than from the left path. Fig. 3.b shows the responses when light impinges just into the left receptor. The three ganglion cells offer three different outputs, as it was suggested previously. Different results should be obtained for light going into the right receptor. In this case, ganglion cell $\mathrm{G}_{3}$, corresponding to photoreceptor $R_{2}$, gives no signal at all: it remains in level "0". On the contrary, $G_{1}$ and $G_{2}$ outputs are constant trains of pulses. These signals are equivalent to a burst of action potentials. The output interval where a periodic train of pulses appears, was obtained in the way reported in ${ }^{2}$. These results are the same than if no signal is detected by the receptors or if the same signals simultaneously reach both channels. 
A symmetric behavior can be obtained if some interconnections are changed. As it was pointed out, there are four possible inputs to each OPLC. Hence, we have the possibility to modify their outputs by adopting the internal adequate configuration as well as modifying the input gate to previous layers, either the two usual OPLC inputs or the control gates.

To obtain the above mentioned behavior, no modifications are made in receptor and horizontal cells. Bipolar cells are now slightly modified. Signals coming from receptor and horizontal layers, after being added, are control signals as before. But one of the two constant inputs appearing in the previous configuration is removed.

The next modification appears at the amacrine level. Signals from upper layers were added, in the previous configuration, before going into the OPLC. On the contrary, both inputs channels are not added now externally. The internal structure of the OPLC do it. The amacrine block is the only one with more than one input. The main reason for this situation is the signal pre-processing action performed by the amacrine. It is present in our model as a delay applied before the signal reaches the OPLC.

This new structure gives different results than in previous configurations. If light goes into the left receptor, signals at ganglion level are different from the asymmetrical case. Ganglion cell at the photoreceptor with light row, shows an output composed by a single peak when the light is switched off. Ganglion cell on the opposite side, gives a train of pulses while the light is on. Finally, the ganglion cell in the middle remains at level "1" when there is no light and presents a burst of pulses while the light is on. This behavior is symmetrical with respect to the input channels.

The last case to be reported corresponds to light signals arriving to $R_{1}$ and $R_{2}$ at different times but with some common interval. Ganglion $G_{1}$ gives pulses during the time $R_{2}$ gets light and $R_{1}$ is in the darkness. Moreover, an additional single pulse is obtained when the light switches off. $G_{3}$ has a train of pulses while there is light at $R_{2}$ and no light at $R_{1}$. Behavior of ganglion $\mathrm{G}_{2}$ is much more complex than of the previous cells. Outputs are trains of action potentials when there is light on either one of the input channels and no light at the other one. Moreover, when there is light at both channels, output is a logic " 0 " and it is a " 1 " when there is no light at the two receptors.

\section{OTHER RESULTS FROM THE PROPOSED SYSTEM}

In the same way than some parts of the mammalian retina has been emulated by the reported structure, some other functions performed at higher levels of the visual cortex, can be obtained too. We will present in this work a proposal to analyze some characteristics of a particular image as, for instance, its symmetry. This kind of analysis is obtained at the visual cortex in most of the vertebrates and it constitutes a very different type of interpretation because has inside a higher level of complexity.

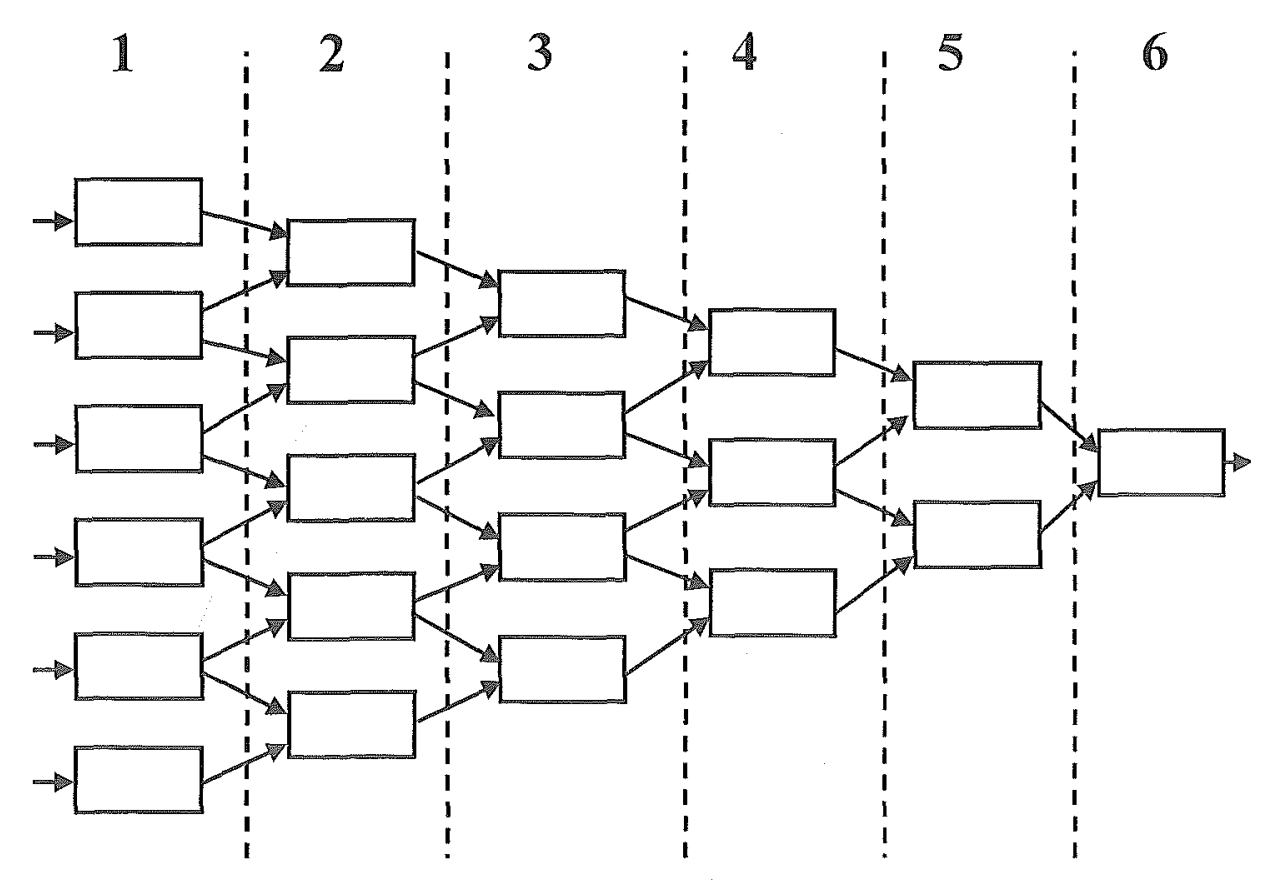

Fig. 4. First layers model of the primary visual cortex. 
The problem to be analyzed corresponds to a situation where some details of an object have a certain type of symmetry. In our present solution just a one-dimensional line will be analyzed. Extensions to higher dimensions are easy to implement. As an example of this case we can give the following one: two columns supporting a frontispiece. If the two columns have the thickness, a linear scan at any level will give a symmetric situation with respect to the columns. If they have different thickness, an asymmetric situation will be present.

In order to accomplish the abovementioned objective, a new structure, as the one shown in Fig. 4, has been adopted. The blocks are again of the same type, OPLCs, than before. The logic functions to be performed by them depend on the type of interpretation it is wanted. As a first example, if the property is desired to know is the symmetry of the light impinging onto the cells, the function to be performed by the OPLCs are simply XOR functions. Some examples are given in Fig 5. Light on a receptor gives a logic " 1 " and no-light gives a " 0 ". In Fig. 5.a, a symmetric light

$\begin{array}{cc}100001 & 110011 \\ 10001 & 01010 \\ 1001 & 1111 \\ 101 & 000 \\ 11 & 00 \\ 0 & 0\end{array}$

(a) Symmmetry: A logic " 0 " is always obtained at the 6th layer

$\begin{array}{ccc}100000 & 111001 & 110001 \\ 10000 & 00101 & 01001 \\ 1000 & 0111 & 1101 \\ 100 & 100 & 011 \\ 10 & 10 & 10 \\ 1 & 1 & 1\end{array}$

(b) Asymmetry: A logic " 1 " is always obtained at the 6th layer

Fig. 5. Results obtained from Fig. 4 for different cases of the incident light symmetry. acts on the first row of cells. In this case, the obtained logic digit gives the results in higher levels of the system. As it can be seen, depending on the order of the symmetry, just zeros are obtained from a certain level on, the fifth row in the first case and the third row in the second one. On the contrary, when the light signal is asymmetric, as in Fig. 5.b, a logic " 1 " is always obtained at the fifth level of the processing layers. This study can be extended to obtain some other properties of the incident light.

\section{ACKNOWLEDGEMENTS}

This work was partly supported by CICYT, grant TIC95-0118 and CAM, grant 07T/0017/1997.

\section{REFERENCES}

1. J. E. Dowling, "The Retina: An Approachable Part of the Brain". The Belknap Press of Harvard University Press. Cambridge, MA (1987).

2. A. González-Marcos and J.A. Martín-Pereda, "Digital chaotic output from an optically processing element", Opt. Eng. 35, 525-535 (1996).

3. J.A. Martín-Pereda and A. González-Marcos: "Some Connections between Neurophysiology and Optical Computing based on the Theory of Complexity". In "Fluctuation phenomena: Disorder and Nonlinearity". Eds.: J. Bishop \& L. Vázquez. World Scientific Press. Singapur. 107-113 (1995)

4. J.A. Martín-Pereda, A. González-Marcos and C. Sánchez-Guillén, "A model of the Mammalian Retina and the Visual Cortex. Disorders of vision". In IEEE Conference on Engineering in Medicine and Biology. Technical Digest CD-ROM 138-140. Amsterdam (1996). 